\title{
Isolated versus Drug Combined Vestibular Rehabilitation for the Treatment of Metabolic Dizziness
}

\author{
Bárbara Vieira Coró ${ }^{*}$ (D), Miguel Angelo Hyppolito² \\ ${ }^{1}$ Medical School of Ribeirão Preto, University of São Paulo, São Paulo, Brazil \\ ${ }^{2}$ Department of Ophthalmology, Otorhinolaryngology and Head and Neck Surgery, Ribeirão Preto Medical School, University of \\ São Paulo, São Paulo, Brazil \\ Email: *ba.vcoro@gmail.com
}

How to cite this paper: Coró, B.V. and Hyppolito, M.A. (2020) Isolated versus Drug Combined Vestibular Rehabilitation for the Treatment of Metabolic Dizziness. International Journal of Clinical Medicine, 11, 111-118.

https://doi.org/10.4236/ijcm.2020.113011

Received: January 11, 2020

Accepted: February 29, 2020

Published: March 3, 2020

Copyright $\odot 2020$ by author(s) and Scientific Research Publishing Inc. This work is licensed under the Creative Commons Attribution International License (CC BY 4.0).

http://creativecommons.org/licenses/by/4.0/

\begin{abstract}
Introduction: Systemic processes and inappropriate use of medications may affect vestibular adaptation. Objectives: To assess the efficacy of isolated and combined drug therapy for treating metabolic dizziness and to determine the effect of multidrug treatment. Methodology: We analyzed 100 questionnaires of patients diagnosed with dizziness of metabolic origin. Results: The group treated with vestibular rehabilitation alone showed improvement with 9 months of therapy $(\mathrm{p}=0.01)$. The group treated with vestibular rehabilitation and medication showed improvement with 45 days of therapy $(\mathrm{p}=0.01)$. The group treated with a single type of drug did not show significant improvement. The group treated with several drugs showed improvement with 45 days of therapy $(p=0.03)$. Conclusion: Combined vestibular rehabilitation resulted in immediate improvement of symptoms without contributing to long-term compensation. Vestibular rehabilitation alone resulted in improvement of symptoms over time. Multidrug treatment was beneficial for immediate relief of dizziness.
\end{abstract}

\section{Keywords}

Dizziness, Metabolism, Vestibular Rehabilitation

\section{Introduction}

Metabolic dysfunctions are considered responsible for labyrinthine changes [1]. Metabolic disorders may act as the main etiological factor in vestibular dysfunction or as an aggravating factor in preexisting vestibular disease [2].

The inner ear is distinguished by its intense metabolic activity; however, it has 
no energy reserve. Small glycemic variations influence its function, causing changes in balance. Metabolic changes in the inner ear cause displacement of potassium from endolymph to perilymph; sodium is concomitantly displaced in the opposite direction. This mechanism causes vertigo, tinnitus, hypoacusis, and auricular plenitude [3].

Vestibular rehabilitation, indicated for treating metabolic dizziness, is influenced by comorbidities in the final response to treatment. In these cases, the objective is integrated otoneurologic therapy with the goal of resolving etiologic factors; this involves rational use of antivertiginous drugs and the application of individualized therapeutic techniques to reduce patient symptoms.

Anti-vertigo medication is currently used as part of combined otoneurologic therapy; however, many researchers caution against the use of multidrug treatments that may cause impairment in vestibular compensation through unintended drug interactions [4]. Thus, our study was undertaken to analyze the outcome of treatment of dizziness of metabolic origin with vestibular rehabilitation in isolation, compared with vestibular rehabilitation combined with medications, and to evaluate the effect of multidrug treatment on the final outcome of vestibular rehabilitation.

\section{Materials and Methods}

This retrospective, observational study was performed by using questionnaire analysis. Data were collected from internal questionnaires administered to patients who attended the vestibular rehabilitation clinic. Only questionnaires of patients with a medical diagnosis of dizziness of metabolic origin were included in the study; questionnaires with incomplete information were excluded from the study. The diagnosis of metabolic dizziness was carried out by the outpatient medical team composed of otolaryngologists. The questionnaires used were designed according to the clinical experience of the researchers, addressing signs and symptoms involved in dizzying crises, social characteristics of the patients, medications in use and objective questions to assess the effectiveness of vestibular rehabilitation therapy.

A total of 642 questionnaires completed between 2002 and 2014 were evaluated; to generate the sample for analysis, 100 questionnaires were randomly selected.

To assess the outcome of vestibular rehabilitation, the response of each patient upon return to therapy was considered. Responses were either "improvement," "worsening", or "maintenance" of dizziness. Five service sessions were evaluated: 30 days, 45 days, 3 months, 6 months, and 9 months after beginning rehabilitation.

\section{Results and Analysis}

We adopted a significance level of $\mathrm{p}<0.05$ in all analyses. We used parametric methods because all variables exhibited a normal distribution (Kolmogorov-Smir- 
nov test). Fisher's exact test for non-paired samples was used to compare groups. For intra-group analyses at different times of therapy, Fisher's exact test was used.

The sample was composed of 100 questionnaires. The age distribution of subjects according to gender (mean and standard deviation) is described in Table 1.

The number of subjects with a diagnosis of dizziness of metabolic origin who also exhibited a metabolic disease was $55 \%(\mathrm{~N}=55)$ in the population studied; metabolic alterations reported were diabetes (28\%), hypothyroidism (24\%), and cholesterol (18\%); $15 \%$ of subjects had more than one metabolic alteration.

Figure 1 shows the drugs used by the study population. A total of $80 \%(\mathrm{~N}=$ $80)$ of the subjects used medication; $62 \%(\mathrm{~N}=62)$ of the subjects underwent combination therapy with two or more pharmacological agents.

The group using medication was composed of 81 patients; 70 of these patients returned for further vestibular rehabilitation. The non-medicated group comprised 19 patients; 14 of these patients returned for further vestibular rehabilitation.

The group treated with vestibular rehabilitation alone exhibited a significant reduction in the symptom of dizziness at the 9-month follow-up $(\mathrm{p}=0.01)$ (Table 2 ). The group treated with vestibular rehabilitation and medication showed a significant reduction in dizziness at the 45-day follow-up $(\mathrm{p}=0.01)$. In a comparison between groups, there was a significant reduction in dizziness at the 9-month follow-up ( $p=0.01)$ for the group treated with vestibular rehabilitation and medication (Table 3).

Notably, 19 patients were using only one type of drug; 18 of these patients returned for further vestibular rehabilitation. Sixty-two patients were using two or more types of drugs; 52 of these patients returned for further vestibular rehabilitation.

The group using one type of drug did not exhibit a significant reduction in dizziness. The group using several drugs showed a significant improvement at the 45-day follow-up $(\mathrm{p}=0.03)$ (Table 4$)$. There was no statistically significant difference between the groups (Table 5 ).

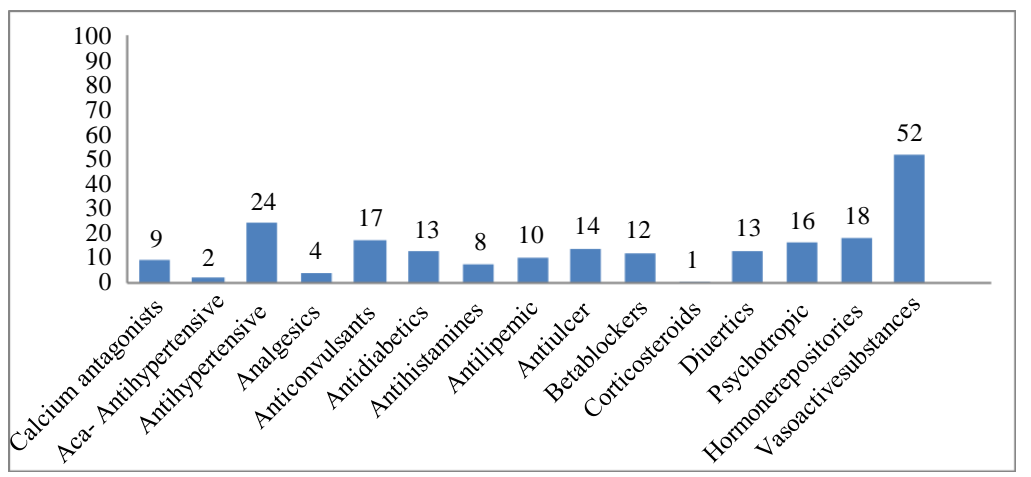

ACa Antihypertensive $=$ Antihypertensive calcium antagonists .

Figure 1. Pharmacological distribution according to its occurrence in the study population. 
Table 1. Age distribution of subjects according to gender: mean and standard deviation.

\begin{tabular}{cccccc}
\hline Gender & $\mathrm{N}$ & $\%$ & Mean Age & Standard Deviation & Coefficient of Variation \\
\hline Female & 69 & $69 \%$ & 52.72 & 16.97 & 32.18 \\
Male & 31 & $31 \%$ & 55.83 & 15 & 26.86 \\
Total & 100 & $100 \%$ & 53.69 & 16.31 & 30.3 \\
\hline
\end{tabular}

Table 2. Analysis of vestibular rehabilitation outcome according to follow-up time in groups with and without medication.

\begin{tabular}{|c|c|c|c|c|c|c|c|c|}
\hline & \multicolumn{2}{|c|}{30 days vs. 45 days } & \multicolumn{2}{|c|}{30 days vs. 3 months } & \multicolumn{2}{|c|}{30 days vs. 6 months } & \multicolumn{2}{|c|}{30 days vs. 9 months } \\
\hline & \multicolumn{2}{|c|}{$\mathrm{N}$} & \multicolumn{2}{|c|}{$\mathrm{N}$} & \multicolumn{2}{|c|}{$\mathrm{N}$} & \multicolumn{2}{|c|}{$\mathrm{N}$} \\
\hline Improvement & 8 & 8 & 8 & 7 & 8 & 10 & 8 & 12 \\
\hline No reply & 6 & 6 & 6 & 7 & 6 & 4 & 6 & 0 \\
\hline $\mathrm{P}$ & \multicolumn{2}{|c|}{1.2964} & \multicolumn{2}{|c|}{1} & \multicolumn{2}{|c|}{0.6946} & \multicolumn{2}{|c|}{$0.0171^{*}$} \\
\hline \multicolumn{9}{|l|}{ G2 } \\
\hline Improvement & 37 & 49 & 37 & 31 & 37 & 31 & 37 & 32 \\
\hline No reply & 33 & 17 & 33 & 23 & 33 & 28 & 33 & 17 \\
\hline $\mathrm{P}$ & \multicolumn{2}{|c|}{$0.0127^{*}$} & \multicolumn{2}{|c|}{0.7163} & \multicolumn{2}{|c|}{1} & \multicolumn{2}{|c|}{0.1918} \\
\hline
\end{tabular}

Legend: G1 = Group without use of medication; G2 = Group using medication. Fisher exact test.

Table 3. Comparison between groups with and without medication according to the outcome of vestibular rehabilitation.

\begin{tabular}{|c|c|c|c|c|c|c|c|c|c|c|}
\hline & \multicolumn{2}{|c|}{30 days } & \multicolumn{2}{|c|}{45 days } & \multicolumn{2}{|c|}{3 months } & \multicolumn{2}{|c|}{6 months } & \multicolumn{2}{|c|}{9 months } \\
\hline & \multicolumn{2}{|c|}{ G1 vs. G2 } & \multicolumn{2}{|c|}{ G1 vs. G2 } & \multicolumn{2}{|c|}{ G1 vs. G2 } & \multicolumn{2}{|c|}{ G1 vs. G2 } & \multicolumn{2}{|c|}{ G1 vs. G2 } \\
\hline & \multicolumn{2}{|c|}{$\mathrm{N}$} & \multicolumn{2}{|c|}{$\mathrm{N}$} & \multicolumn{2}{|c|}{$\mathrm{N}$} & \multicolumn{2}{|c|}{$\mathrm{N}$} & \multicolumn{2}{|c|}{$\mathrm{N}$} \\
\hline Improvement & 8 & 37 & 8 & 49 & 7 & 31 & 10 & 31 & 12 & 32 \\
\hline No reply & 6 & 33 & 6 & 17 & 7 & 28 & 4 & 23 & 0 & 17 \\
\hline $\mathrm{P}$ & \multicolumn{2}{|c|}{1} & \multicolumn{2}{|c|}{0.2104} & \multicolumn{2}{|c|}{1} & \multicolumn{2}{|c|}{0.379} & \multicolumn{2}{|c|}{$0.0142^{*}$} \\
\hline
\end{tabular}

Legend: G1 = Group without use of medication; G2 = Group using medication. Fisher exact test.

Table 4. Analysis of vestibular rehabilitation outcomes according to follow-up times in groups with a single type of medication and with multiple medications.

\begin{tabular}{|c|c|c|c|c|c|c|c|c|}
\hline & \multicolumn{2}{|c|}{30 days vs. 45 days } & \multicolumn{2}{|c|}{30 days vs. 3 months } & \multicolumn{2}{|c|}{30 days vs. 6 months } & \multicolumn{2}{|c|}{30 days vs. 9 months } \\
\hline & \multicolumn{2}{|c|}{$\mathrm{N}$} & \multicolumn{2}{|c|}{$\mathrm{N}$} & \multicolumn{2}{|c|}{$\mathrm{N}$} & \multicolumn{2}{|c|}{$\mathrm{N}$} \\
\hline Improvement & 8 & 11 & 8 & 7 & 8 & 9 & 8 & 12 \\
\hline No reply & 10 & 5 & 10 & 7 & 10 & 3 & 10 & 0 \\
\hline $\mathrm{P}$ & \multicolumn{2}{|c|}{0.1854} & \multicolumn{2}{|c|}{1} & \multicolumn{2}{|c|}{0.1414} & \multicolumn{2}{|c|}{0.2543} \\
\hline \multicolumn{9}{|l|}{ G2 } \\
\hline Improvement & 29 & 38 & 29 & 24 & 29 & 22 & 29 & 25 \\
\hline No reply & 23 & 12 & 23 & 21 & 23 & 20 & 23 & 14 \\
\hline $\mathrm{P}$ & \multicolumn{2}{|c|}{$0.0382^{*}$} & \multicolumn{2}{|c|}{0.8403} & \multicolumn{2}{|c|}{0.8358} & \multicolumn{2}{|c|}{0.5189} \\
\hline
\end{tabular}

Legend: G1 = Group with single medication; G2 = Group with multiple medications. Fisher exact test. 
Table 5. Comparison between groups using one and more types of medications with the result of vestibular rehabilitation.

\begin{tabular}{|c|c|c|c|c|c|c|c|c|c|c|}
\hline & \multicolumn{2}{|c|}{30 days } & \multicolumn{2}{|c|}{45 days } & \multicolumn{2}{|c|}{3 months } & \multicolumn{2}{|c|}{6 months } & \multicolumn{2}{|c|}{9 months } \\
\hline & \multicolumn{2}{|c|}{ G1 vs. G2 } & \multicolumn{2}{|c|}{ G1 vs. G2 } & \multicolumn{2}{|c|}{ G1 vs. G2 } & \multicolumn{2}{|c|}{ G1 vs. G2 } & \multicolumn{2}{|c|}{ G1 vs. G2 } \\
\hline & \multicolumn{2}{|c|}{$\mathrm{N}$} & \multicolumn{2}{|c|}{$\mathrm{N}$} & \multicolumn{2}{|c|}{$\mathrm{N}$} & \multicolumn{2}{|c|}{$\mathrm{N}$} & \multicolumn{2}{|c|}{$\mathrm{N}$} \\
\hline Improvement & 29 & 8 & 38 & 11 & 24 & 7 & 22 & 9 & 25 & 7 \\
\hline No reply & 23 & 10 & 12 & 5 & 21 & 7 & 20 & 3 & 14 & 3 \\
\hline $\mathrm{P}$ & \multicolumn{2}{|c|}{0.4272} & \multicolumn{2}{|c|}{0.7433} & \multicolumn{2}{|c|}{1} & \multicolumn{2}{|c|}{0.2003} & \multicolumn{2}{|c|}{1} \\
\hline
\end{tabular}

Legend: G1 = Group with single medication; G2 = Group with multiple medications. Fisher exact test.

\section{Discussion}

The distribution of the sample regarding gender and average age (Table 1) showed that dizziness was prevalent in female patients in this study, consistent with prior literature [5] [6]. Women seek medical care more frequently than men, regardless of factors such as the variation of the monthly hormonal cycle and menopause, which exhibits dizziness as a primary symptom [7] [8].

Anti-vertigo drugs are used as vestibular rehabilitation allies for treating dizziness; however, many professionals are concerned with the side effects described in the literature, among them impairment in vestibular compensation.

We sought to characterize the vestibular rehabilitation outcome by using an isolated and combined approach with medications, and to analyze the effect of the multidrug treatment in subjects who used two or more types of drugs.

We analyzed a group of individuals using medication and another group without; however, both performed vestibular rehabilitation. In the analysis between groups, there was a significant improvement in dizziness with 9 months of treatment for the group using drugs combined with therapy. For better understanding, intra-group analysis was performed, which indicated that this time effect was attributed to vestibular rehabilitation therapy.

The effect of the combined treatment of vestibular rehabilitation and medications was significant for relieving symptoms of dizziness at 45 days of follow-up, but the improvement did not remain significant over time. This indicates that the drug helps in immediate reduction of symptoms, but does not contribute to long-term compensation. We verified that vestibular rehabilitation as an isolated treatment resulted in a significant improvement in symptom of dizziness at 9 months of follow-up, indicating that it is an effective treatment and has an effect over time.

Snifer et al. (2004) [9] evaluated the response of patients with vestibular disorder to treatment with vestibular rehabilitation and medication, both combined and in isolation. They found that patients treated with vestibular rehabilitation alone, or in combination with medication, showed clinical improvement and significant differences in the Dizziness Handicap Inventory. The group treated with medication alone did not show clinical improvement and or a significant difference in the Dizziness Handicap Inventory. In that study, the use of a single vasodilator drug was evaluated. 
The works of Shoair et al. (2011) [10] and Pinheiro et al. (2013) [11] concluded that multidrug treatment is associated with increased risk of dizziness and injury in vestibular compensation. For the treatment of vertigo of vestibular origin, two or more types of drugs are used because there is no specific drug that acts on the vestibular system [12].

Considering the drug interactions and various side effects, among them the increased of dizziness, we sought to characterize the difference between the group using a single type of medication and another group using multiple drugs. We found that the group using a single type of medication did not show significant improvement in dizziness during the time period of the study. The group with drug interactions showed significant improvement in dizziness after 45 days of treatment.

The use of drug interactions was beneficial with an immediate effect for the relief of dizziness; however, a long-term effect was not observed.

Although drug interactions do not contribute to long-term central compensation, in this study it was possible to identify a positive action at the beginning of treatment, with a reduction in symptoms, which is necessary because the patient benefits from the immediate relief of dizziness to initiate the vestibular rehabilitation exercises. Caution should be taken to indicate the correct dosage of drugs and the time of use.

The factors that interfere in the evolution of cases of metabolic vestibulopathy and the success of short-term vestibular rehabilitation are inadequate eating habits, sedentary lifestyle, sleep disturbances, delay in the control of underlying disease and, in the case of women, hormonal variations and menopause. Other factors, such as failure to perform proposed exercises and/or inadequate performance, are common during vestibular rehabilitation, regardless of the etiology of dizziness, and also occurred in the study population.

\section{Conclusions}

Vestibular rehabilitation combined with medication for the treatment of dizziness resulted in immediate improvement of dizziness symptoms, and did not contribute to long-term compensation. Vestibular rehabilitation alone in the treatment of dizziness resulted in improvement of symptoms over time.

Multidrug treatment was beneficial for immediate relief of dizziness but did not contribute to long-term compensation.

The use of medications aids in early stages of rehabilitation of disorders of the peripheral vestibular system, which are complemented and sedimented by vestibular rehabilitation exercises.

\section{Acknowledgements}

We thank the Speech Therapist Ana Paula do Rego André, responsible for the Vestibular Rehabilitation outpatient clinic, for allowing our group access to the questionnaires and supporting the completion of this work. 


\section{Ethical Standards}

This study was approved by the ethics council of the authors' institution. In this study, we followed the norms advocated by the ethics and research commission with respect to human research, ethical secrecy, and biosafety norms.

Only the researchers had access to the information provided by the questionnaires.

The authors state that all procedures that contributed to this work complied with the ethical standards of the relevant national and institutional guidelines on human experimentation, and with the Declaration of Helsinki of 1975, as revised in 2008 .

\section{Conflicts of Interest}

The authors declare no conflicts of interest regarding the publication of this paper.

\section{References}

[1] Scherer, L.P. and Lobo, M.B. (2002) Search of the Nystagmus/Positional Vertigo and Electronystagmography Evaluation in a Group of Diabetics Mellitus Type I. Revista Brasileira de Otorrinolaringologia, 68, 355-360. https://doi.org/10.1590/S0034-72992002000300010

[2] Serra, A.P., Lopes, K.C., Dorigueto, R.S. and Ganança, F.F. (2009) Blood Glucose and Insulin Levels in Patients with Peripheral Vestibular Disease. Brazilian Journal of Otorhinolaryngology, 75, 701-705.

https://doi.org/10.1590/S1808-86942009000500015

[3] Ferreira, J.C.A., Guimaraes, R.E.S., Becker, H.M.G., Gonçalves, T.M.L., Silva, C.D.L., Crosara, P.F.T., et al. (2000) Metabolic Evaluation of the Patient with Labyrinth Disease. Arquivos internacionais de otorrinolaringologia, 4, 28-32.

[4] Costa, F.M., Doná, F. and Paulino, C.A. (2013) Drug Interactions in Elderly Woman with Vestibular Disorder: A Case Report. Revista Equilíbrio Corporal e Saúde, 5, 49-59.

[5] Nishino, L.K., Ganança, C.F., Manso, A., Campo, C.A.H. and Korn, G.P. (2005) Personalized Vestibular Rehabilitation: Medical Chart Survey with Patients Seen at the Ambulatory of Otoneurology of I.S.C.M.S.P. Revista Brasileira de Otorrinolaringologia, 71, 440-407. https://doi.org/10.1590/S0034-72992005000400007

[6] Neuhauser, H.K., von Brevern, M., Radtke, A., Lezius, F., Feldmann, M., Ziese, T., et al. (2005) Epidemiology of Vestibular Vertigo: A Neurotologic Survey of the General Population. Neurology, 65, 898-904. https://doi.org/10.1212/01.wnl.0000175987.59991.3d

[7] Bittar, R.S.M. (1997) Hormone Labyrinth: Hormone Steroids, Estrogen and Progesterone. Arquivos Internacionais de Otorrinolaringologia, 4, 32.

[8] Bittar, R.S.M., Oticica, J., Bottino, M.A., Ganança, F.F. and Dimitrov, R. (2013) Population Epidemiological Study on the Prevalence of Dizziness in the City of São Paulo. Brazilian Journal of Otorhinolaryngology, 79, 688-698. https://doi.org/10.5935/1808-8694.20130127

[9] Sznifer, J., Bitter, R.M.S., Pedalini, M.E.B., Lorenzi, M.C. and Sanchez, T.G. (2004) 
Balance Disturbances of Vascular Origin: Medication or Vestibular Rehabilitation? International Archieves of Otorhinolaryngology, 8, 134-141.

[10] Shoair, O.A., Nyandege, A.N. and Slattum, P.W. (2011) Medication-Related Dizziness in the Older Adult. Otolaryngologic Clinics of North America, 44, 455-471. https://doi.org/10.1016/j.otc.2011.01.014

[11] Pinheiro, J.S., Carvalho, M.F.C. and Luppi, G. (2013) Drug Interaction and Pharmacotherapy of Geriatric Patients with Dementia. Revista Brasileira de Geriatria e Gerontologia, 12, 303-314. https://doi.org/10.1590/S1809-98232013000200010

[12] Claussen, C.F. and Constantinescu, L. (1995) Mode of Action of Anti Vertiginous Drugs. Proceedings of the NES, 21, 65-72. 